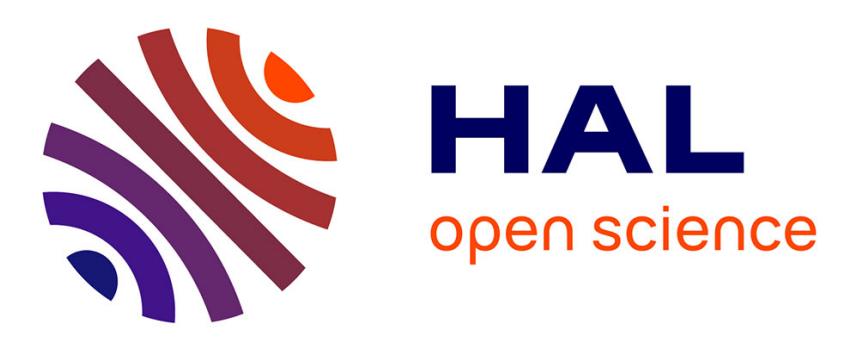

\title{
Résistance au champ des blés en semis d'automne au virus de la mosaïque du blé (wheat soil-borne mosaic virus)
}

Hervé Lapierre, Michel Courtillot, Chantal Kusiak, Djabbar Hariri

\section{- To cite this version:}

Hervé Lapierre, Michel Courtillot, Chantal Kusiak, Djabbar Hariri. Résistance au champ des blés en semis d'automne au virus de la mosaïque du blé (wheat soil-borne mosaic virus). Agronomie, 1985, 5 (7), pp.565-572. hal-00884785

\section{HAL Id: hal-00884785 https://hal.science/hal-00884785}

Submitted on 1 Jan 1985

HAL is a multi-disciplinary open access archive for the deposit and dissemination of scientific research documents, whether they are published or not. The documents may come from teaching and research institutions in France or abroad, or from public or private research centers.
L'archive ouverte pluridisciplinaire HAL, est destinée au dépôt et à la diffusion de documents scientifiques de niveau recherche, publiés ou non, émanant des établissements d'enseignement et de recherche français ou étrangers, des laboratoires publics ou privés. 


\title{
Résistance au champ des blés en semis d'automne au virus de la mosaïque du blé (wheat soil-borne mosaic virus)
}

\author{
Hervé LAPIERRE, Michel COURTILLOT, Chantal KUSIAK \& Djabbar HARIRI
}

I.N.R.A., Station de Pathologie végétale, Route de St-Cyr, F 78000 Versailles

RÉSUMÉ

Le comportement au champ de 57 cultivars de blés vis-à-vis du virus de la mosaïque du blé (VMB) ou wheat soil borne mosaic virus a permis de caractériser 13 cultivars résistants.

La recherche de cystosores de Polymyxa dans les racines et celle de l'antigène viral (ELISA sandwich) dans les jeunes talles à la fin du printemps ont été effectuées chez ces cultivars sans symptôme. Les résultats obtenus sont les suivants :

- Deux cultivars contiennent une faible concentration en virus : (26.3.10) et "Capitole "; Polymyxa est fréquent chez "Capitole » et n'a pas été trouvé chez (26.3.10). Ce dernier cultivar peut être considéré comme présentant une bonne résistance au Polymyxa.

- Pour 10 cultivars, la concentration de l'antigène viral est très faible ou nulle. Ces cultivars peuvent être rangés en 3 catégories :

a. Chez « Cargo », l'absence de cystosores dans les racines et la non-détection du virus dans les feuilles peuvent être considérées comme l'expression d'une résistance probable à l'installation du vecteur présumé du VMB.

b. Chez "Cocagne », " Fidel », « Fleuron », (CWW 1335/2), la résistance à la maladie peut être expliquée par l'activité de deux processus dont l'importance relative reste à préciser : une résistance partielle au Polymyxa, associée à une résistance partielle au virus.

c. Chez « Tomclair », « Sabre », « Avalon », « Apexal », « Albatros », en raison de l'abondance des cystosores dans les racines, la résistance à la maladie peut être attribuée à une résistance à la multiplication du virus dans la partie aérienne des plantes.

Ce premier tri de 12 cultivars sans symptôme en fonction de leur capacité à multiplier Polymyxa ou le VMB dans la partie aérienne des plantes devrait permettre de poursuivre en particulier deux objectifs : mieux estimer le rôle des différents cultivars de blés dans la constitution de l'inoculum du sol, envisager l'étude des mécanismes de résistance à la maladie virale.

Mots clés additionnels : ELISA, Polymyxa graminis

On the basis of the field behaviour of 57 wheat cultivars in the presence of wheat soil-borne mosaic virus, it was possible to characterize 13 resistant cultivars. Tests for cystosori of Polymyxa graminis in the roots, and for viral antigen (ELISA test) in young tillers, were carried out in late spring in 12 of these symptomless cultivars. Two cultivars, (26.3.10) and "Capitole", contained a low virus concentration. P. graminis frequently occurred in "Capitole" and was not found in (26.3.10), which may be regarded as adequately resistant to $P$. graminis.

The other 10 cultivars showed little or no viral antigen concentration, and could be divided into 3 categories : 1) in "Cargo", the absence of cystosori in the roots and non-detection of virus in the leaves was probably due to resistance to the suspected vector of WSBMV ; 2) in "Cocagne", "Fidel", "Fleuron" and (CWW 1335/2), disease resistance may be explained by the action of two processes whose relative importance needs to be determined, i.e. partial resistance to $P$. graminis, associated with partial resistance to the virus ; 3 ) in "Tomclair", "Sabre", "Avalon", "Apexal" and "Albatros" which show abundant cystosori in the roots, disease resistance may originate from resistance to virus multiplication in the plant shoots. This preliminary selection of 12 symptomless cultivars based on their ability to multiply $P$. graminis or WSBMV in the shoots opens two possibilities : assessment of the contribution of the various wheat cultivars to soil inoculum and more thorough study of the mechanisms of resistance to the virus.

Additional key words : ELISA, Polymyxa graminis 


\section{INTRODUCTION}

Le virus de la mosaïque du blé (VMB) ou wheat soil borne mosaic virus a été décrit depuis très longtemps en Amérique du Nord et en Asie. Ce virus à génome divisé (TSUCHIZAKI et al., 1975) comprend 2 particules tubulaires et quelquefois une $3^{\mathrm{e}}$ provenant d'une délétion de la $2^{\mathrm{e}}$ (SHIRAKO \& BRAKKE, 1984). Ce virus est considéré comme un membre possible des Tobamovirus (Powell, 1976; MATTHEWS, 1982) sans qu'aucune confirmation définitive n'ait été apportée jusque-là.

Aux U.S.A., ces dernières années, les surfaces contaminées par le VMB semblent avoir pris une extension considérable (BOCKUS \& NiBLETT, 1984). Ce virus a été identifié plus récemment en Europe : avec certitude en Italie (CANOVA, 1962; LANGENBERG comm. pers.), comme probable en République Démocratique Allemande (PROESELER et al., 1982) et en U.R.S.S. (cité par PROESELER et al., 1982). Son existence en Turquie a été envisagée (KURCMAN, 1981).

En France, une souche de type symptomatologique " mosaique jaune » de ce virus a été reconnue depuis 1977 (LAPIERRE et al., 1984). Ce virus est actuellement observé sur de grandes surfaces, particulièrement sur le pourtour ouest et sud du Bassin parisien.

Depuis de nombreuses années, des études portant sur la recherche de sources de résistance à ce virus ont été entreprises (Mc KINNEY, 1948). Les composantes génétiques de cette résistance commencent à être précisées (MODAwı et al., 1982) sans que ni les niveaux de cette résistance au vecteur ou/et au virus, ni les mécanismes en cause ne soient connus. En France, des cultivars résistants au champ ont été observés depuis quelques années (LAPIERRE et al., 1984). L'objet de cette étude est d'apporter des éléments d'appréciation sur la capacité de différents cultivars de blé à multiplier au champ le virus et son vecteur probable Polymyxa graminis.

\section{MATÉRIEL ET MÉTHODES}

\section{A. Parcelles expérimentales et cultivars étudiés}

Les prélèvements d'échantillons proviennent de 2 essais variétaux en petites parcelles dans des zones fortement contaminées par le virus et ayant porté au moins un précédent blé. Les deux essais, implantés dans le Loir-et-Cher, l'un à Brévainville (37 cultivars, 2 répétitions), l'autre à Herbault ( 32 cultivars, 2 répétitions) ont permis l'étude de 50 blés tendres et de 7 blés durs. Les cultivars étudiés non inscrits au catalogue français sont présentés entre parenthèses.

\section{B. Détection immunoenzymatique du VMB}

Le sérum utilisé a été obtenu à partir d'un antigène et selon un protocole d'immunisation en partie décrit (LAPIERRE et al., 1984). Les caractéristiques en ELISA sandwich (CLARK \& ADAMS, 1977) de ce sérum sont voisines de celles du sérum qui nous a été aimablement fourni par le Dr BRAKKE (Université du
Nebraska, U.S.A.) : dilution limite du virus purifié $10 \mathrm{ng}$, dilution limite d'un extrait foliaire d'une plante présentant des symptômes $(1 / 10000)$, dilution limite d'un extrait racinaire d'une plante après $7 \mathrm{j}$ d'infection au laboratoire $(1 / 400)$. La technique ELISA (sandwich) est mise en ouvre de la façon suivante : les $\gamma$ globulines contenant les anticorps spécifiques du VMB sont partiellement purifiées par précipitation au sulfate d'ammonium saturé. Leur fixation sur les plaques de microtitration s'effectue à partir d'une solution à la concentration de $1 \mu \mathrm{g} / \mathrm{ml}(2 \mathrm{~h}$ d'incubation à $33^{\circ} \mathrm{C}$ ). Après $16 \mathrm{~h}$ à $4{ }^{\circ} \mathrm{C}$, les extraits de plante sont éliminés des alvéoles des plaques de microtitration et remplacés par une solution de $\gamma$ globulines couplées à la phosphatase alcaline à la concentration de $2 \mu \mathrm{g} / \mathrm{ml}$ et maintenus $5 \mathrm{~h}$ à $33^{\circ} \mathrm{C}$.

La révélation de l'enzyme fixé est effectuée par le p. nitrophénylphosphate $(1 \mathrm{mg} / \mathrm{ml})$ sur une période de $1 \mathrm{~h}$ à $24 \mathrm{~h}$ suivant les essais. Les plaques de microtitration sont maintenues à $20^{\circ} \mathrm{C}$ dans des boîtes fermées dont l'ambiance hygrométrique est voisine de 100 p. 100. Les densités optiques (D.O.) exploitées correspondent aux différences de mesures effectuées entre 2 temps d'hydrolyse du substrat. Les D.O. des blancs sans matériel végétal sont retranchées des D.O. des échantillons d'extraits sains ou à analyser. Deux répétitions sont réalisées au niveau de la plaque de microtitration pour chaque échantillon et 6 pour les témoins sains.

\section{Méthode d'estimation de la fréquence de Polymyxa et de quelques autres organismes dans les racines de blé}

Les fragments racinaires examinés au microscope optique proviennent, pour chaque cultivar, d'au minimum 12 racines appartenant à au moins 4 plantes et sont prélevés sur une épaisseur de sol voisine de $40 \mathrm{~cm}$. Ces fragments sont abondamment lavés à l'eau courante puis à l'eau distillée. On a prélevé sur ces racines et sur leurs ramifications 60 à 100 fragments de 80 à $800 \mu \mathrm{m}$ de diamètre, les parties les plus épaisses étant délaissées. Pour 7 fragments sur 8 , le diamètre est compris entre 120 et $360 \mu \mathrm{m}$, pour la moitié d'entre eux, il est inférieur à environ $210 \mu \mathrm{m}$. La somme des longueurs de ces fragments est d'environ $20 \mathrm{~cm}$. Un $2^{\mathrm{e}}$ lot d'une centaine de fragments provenant d'une dizaine de plantes est examiné si l'on n'a pas trouvé de cystosores de Polymyxa ou si l'on n'en a trouvés de bien caractérisés que sur un seul fragment.

Ces fragments sont colorés par le bleu coton au lactophénol, chauffés jusqu'à légère ébullition et observés dans le lactophénol après ébullition modérée.

Tous les organismes observés dans les racines sont notés, mais les tableaux présentés dans cette étude ne font état que de certains d'entre eux à leurs stades les plus visibles et reconnaissables :

- Cystosores de Polymyxa. Du fait des incertitudes de la systématique actuelle sur les Plasmodiophorales et sur les genres Polymyxa et Ligniera, nous attribuerons les formes de conservation (cystosores) observées au genre Polymyxa sans autre précision.

- Zygosporanges (ou " kystes ») d'Olpidium. 
- Pythium sous forme d'oospores et d'oogones de plus de $6 \mu \mathrm{m}$ de diamètre.

- Nématodes endoracinaires à divers stades.

Les observations de ces échantillons ont été notées selon l'échelle suivante :

0 = organisme non détecté,

1 = très rare : un à quelques éléments dans un seul fragment,

2 = rare: rares éléments dans plusieurs fragments ou assez nombreux éléments dans un seul fragment,

3 = assez rare : éléments abondants dans 2 ou 3 fragments,

4 = peu fréquent abondance dans 4 à 6 fragments, 5 = assez fréquent: abondance dans plus de 6 fragments.

\section{RÉSULTATS}

\section{A. Expression des symptômes de mosaïque}

Un seul critère de sensibilité au VMB a été retenu ici : la fréquence des symptômes de mosaïque observée. Ce type de notation a pour but de faire intervenir la capacité des cultivars à être reconnus par le vecteur du virus et à exprimer des symptômes sans tenir compte de leur gravité. Ces notations sont effectuées sur 200 plantes à 3 époques entre le début du mois de mai et la lïn du mois de juin.

Pour les 57 cultivars notés dans les 2 essais suivis, environ 25 p. 100 n'ont pas présenté de symptômes au champ (tabl. la et 1b). Le cultivar (FD 83043) ne montre de symptômes que sur quelques talles secondaires et constitue le seul élément d'un groupe à très faible fréquence de symptômes. Pour les cultivars de faible sensibilité, moins de 1 p. 100 des talles les plus développées montrent des symptômes de mosaïque. Les talles de taille réduite de ce groupe peuvent être affectées chez plus de 10 p. 100 d'entre elles. Les cultivars de sensibilité modérée, les plus nombreux, mon-

\section{TABLEAU 1a}

Sensibilité des blés au VMB.

Field reaction of winter wheat cultivars to WSBMV.

Blés ne présentant pas de symptômes de mosaïque du blé au champ Localisation : $B=$ BREVAINVILLE $H=$ HERBAULT.

Cultivars without leaf symptoms in the field

Location of the experiment : $B=B R E ́$ VAINVILLE $H=$ HERBAULT.

\begin{tabular}{lccc}
\hline \hline & \multicolumn{2}{c}{$\begin{array}{c}\text { Blés tendres } \\
\text { soft wheat }\end{array}$} & \multicolumn{1}{c}{$\begin{array}{c}\text { Blé dur } \\
\text { durum wheat }\end{array}$} \\
\hline Albatros & Localisation & Localisation \\
Apexal & $\mathrm{H}$ & Tomclair & B \\
Avalon & $\mathrm{H}$ & & \\
Capitole & $\mathrm{H}$ & & \\
Cargo & $\mathrm{H}$ & \\
Cocagne & $\mathrm{B}$ & \\
Fidel & $\mathrm{B}$ & \\
Fleuron & $\mathrm{B}, \mathrm{H}$ & \\
Rempart & $\mathrm{H}$ & \\
Sabre & $\mathrm{H}$ & \\
(CWW 1335/2) & $\mathrm{H}$ & \\
(26.3.10) & $\mathrm{B}$ & \\
\hline
\end{tabular}

TABLEAU $\mathbf{l b}$

Sensibilité des blés au VMB.

Field reaction of winter wheat cultivars to WSBMV.

Blés présentant des symptômes de mosaique du blé au champ.

Entre parenthèses: blés non inscrits au catalogue français.

Cultivars showing various leaf symptoms. In parentheses: wheat cultivars not registered in the French catalogue of varieties.

\begin{tabular}{|c|c|c|c|c|}
\hline Fréquence des symptômes & Blés tendres & Loc. & Blés durs & Loc. \\
\hline \multirow[t]{7}{*}{ Faible } & Aquila & $\mathrm{H}$ & & \\
\hline & Arminda & $\mathrm{H}$ & & \\
\hline & Vizir & $\mathrm{B}, \mathrm{H}$ & & \\
\hline & (FD 74069/201) & B & & \\
\hline & (FD 83038) & B & & \\
\hline & (H 01) & $\mathrm{B}, \mathrm{H}$ & & \\
\hline & (H 12) & $\mathrm{B}, \mathrm{H}$ & & \\
\hline \multirow[t]{15}{*}{ Moyenne } & Aboukir & B & Clairdoc & B \\
\hline & Arcole & B & (Cando) & B \\
\hline & Aubaine & $\mathrm{H}$ & (3919) & $\mathrm{B}$ \\
\hline & Cargimarec & $\mathrm{H}$ & & \\
\hline & Chopin & B & & \\
\hline & Corin & $\mathrm{H}$ & & \\
\hline & Garant & $\mathrm{B}, \mathrm{H}$ & & \\
\hline & Promentin & $\mathrm{B}, \mathrm{H}$ & & \\
\hline & Rotonde & $\mathrm{H}$ & & \\
\hline & Suffren & $\mathrm{B}$ & & \\
\hline & Tenor & $\mathrm{H}$ & & \\
\hline & Thesée & $\mathrm{H}$ & & \\
\hline & Ulm & $\mathrm{H}$ & & \\
\hline & (FD 83322) & B & & \\
\hline & (8115) & B & & \\
\hline \multirow[t]{14}{*}{ Forte } & (Baron) & B & Mondur & $\mathrm{H}$ \\
\hline & Beauchamp & $\mathrm{H}$ & Regal & B \\
\hline & Campremy & $\mathrm{H}$ & (C. 173) & B \\
\hline & Belaviso & $\mathrm{H}$ & & \\
\hline & Festival & $\mathrm{B}, \mathrm{H}$ & & \\
\hline & Fluto & $\mathrm{H}$ & & \\
\hline & Hardi & $\mathrm{B}, \mathrm{H}$ & & \\
\hline & Horace & B & & \\
\hline & Master & $\mathrm{B}, \mathrm{H}$ & & \\
\hline & Mission & $\mathrm{B}, \mathrm{H}$ & & \\
\hline & Pursang & B & & \\
\hline & Scipion & B & & \\
\hline & (FD 83277) & B & & \\
\hline & (57579) & B & & \\
\hline Variable & Talent & $\mathbf{H}$ & & \\
\hline
\end{tabular}

trent des symptômes chez plus de 1 et moins de 10 p. 100 des talles développées. Provisoirement, le cultivar «Talent» n'a pas été inclus dans l'un des groupes ainsi définis, en raison de la variabilité de ses réponses dans différents environnements en présence d'autres cultivars présentant des fréquences reproductibles de symptômes ; le plus souvent, la fréquence des symptômes de ce cultivar le rattache au groupe des blés très sensibles, en particulier dans les 2 essais présentés ici.

\section{B. Détection immunoenzymatique du VMB dans la plante}

\section{Recherche d'un milieu favorisant la mise en évidence du VMB en ELISA}

La difficulté à broyer les racines de plantes développées nous a amenés à analyser l'effet, sur la détection du VMB, de l'azote liquide apporté en vue de réduire 
TABLEAU 2

Effet des différentes concentrations d'urée sur la détection du VMB dans les racines de blé " Hardi » en ELISA. Effect of urea on ELISA detection of WSBMV in roots of wheat cv. "Hardi».

\begin{tabular}{|c|c|c|c|c|c|c|c|c|}
\hline \multirow{4}{*}{$\begin{array}{l}\text { Dilutions des extraits bruts } \\
\text { Dilutions of crude extracts }\end{array}$} & \multicolumn{4}{|c|}{ D.O. à $405 \mathrm{~nm}$} & & & & \\
\hline & \multicolumn{4}{|c|}{$\begin{array}{l}\text { Racines saines } \\
\text { Healthy roots }\end{array}$} & \multicolumn{4}{|c|}{$\begin{array}{l}\text { Racines virosées } \\
\text { Infected roots }\end{array}$} \\
\hline & \multicolumn{8}{|c|}{ Concentration en urée } \\
\hline & 0 & $0,25 \mathrm{M}$ & $0,5 \mathrm{M}$ & M & 0 & $0,25 \mathrm{M}$ & $0,5 \mathrm{M}$ & M \\
\hline $1 / 5$ & 0,645 & 0,549 & 0,641 & 0,411 & 0,474 & 0,873 & 1,571 & 1,891 \\
\hline $1 / 10$ & 0,560 & 0,698 & 0,587 & 0,467 & 0,422 & 0,596 & 1,507 & 1,820 \\
\hline $1 / 30$ & 0,427 & 0,632 & 0,444 & 0,371 & 0,385 & 0,499 & 1,260 & 1,711 \\
\hline $1 / 50$ & 0,381 & 0,581 & 0,380 & 0,374 & 0,333 & 0,430 & 0,882 & 1,365 \\
\hline
\end{tabular}

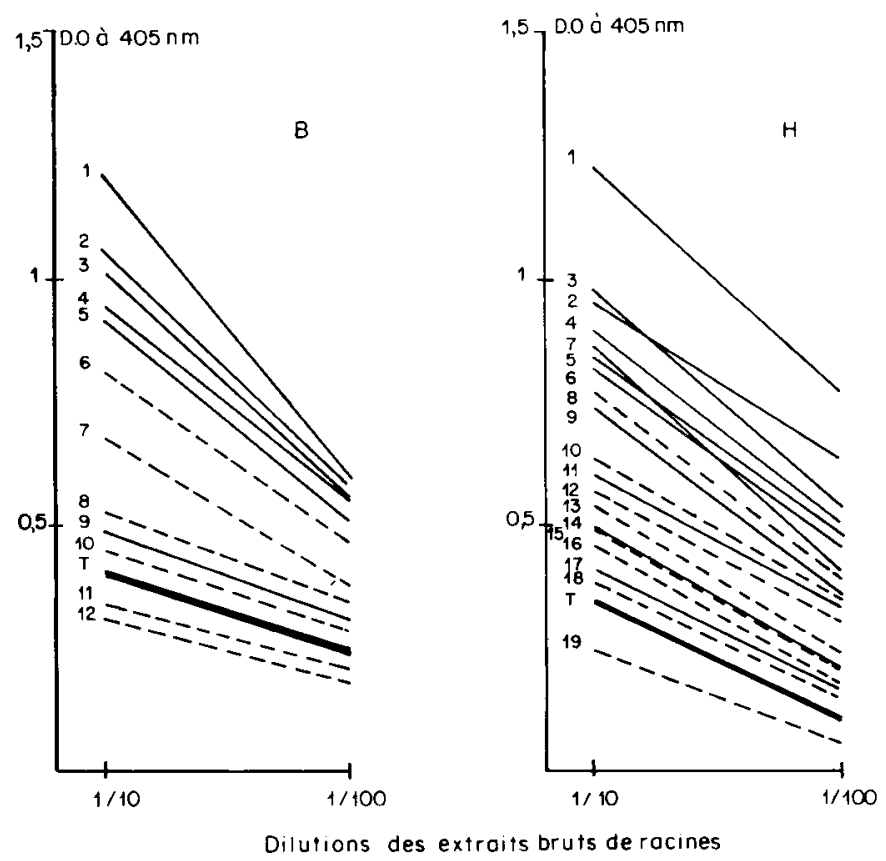

Figure 1

Détection en ELISA du VMB dans les extraits bruts de racines de différents cultivars de blé. Les échantillons sont prélevés à la floraison, soit à Brévainville $(B)$, soit à Herbault $(H)$. Les extraits sont dilués 10 ou 100 fois.

ELISA detection of WSBMV in crude root extracts 10 or 100 times diluted).

B : (1) " Master », (2) " Festival », (3) " Scipion », (4) « Aubaine », (5) " Pursang », (6) "Cargo », (7) "Cocagne », (8) " Fidel ", (9) (FD 83043), (10) (FD 83038), (II) (CWW 1355/2), (12) $(26.3 .10)$.

H:(1) " Master ", (2) “ Aquila », (3) "Fluto », (4) " Festival ", (5) « Arminda », (6) « Aubaine », (7) " Vizir », (8) " Capitole », (9) (H 12), (10) « Albatros », (11) « Hardi », (12) « Avalon ", (13) " Fleuron », (14) " Rempart ", (15) (H 01), (16) « Sabre », (17) « Talent », (18) « Apexal », (19) « Fidel ».

.- Cultivars avec symptômes foliaires; with foliar symptoms. .- - - Cultivars sans symptôme foliaire; without foliar symptoms.

cette partie du végétal à l'état de poudre. Cette technique a dû être rejetée en raison de son effet fortement négatif sur l'antigénicité des particules virales.

Pour éviter l'agrégation des particules virales, l'urée a été utilisée en ELISA, en particulier pour détecter le virus A de la pomme de terre (GugerLI, 1979). Dans le cas du VMB, l'apport au milieu de broyage de con-
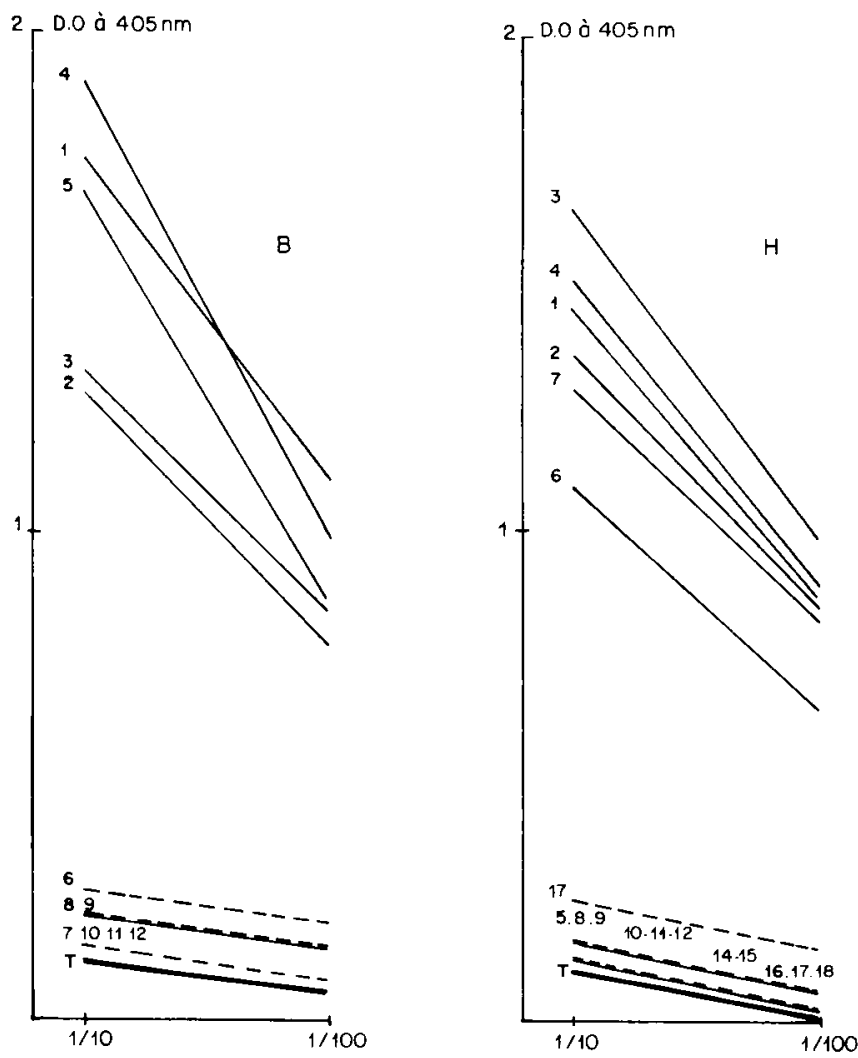

Dilutions des extraits bruts de feuilles

Figure 2

Idem figure 1, dans les extraits bruts de feuilles. As for fig. 1 - leaf extracts.

centrations variables d'urée montre que cette molécule améliore nettement la détection du virus contenu dans les racines (tabl. 2). On observe, par ailleurs, une meilleure reproductibilité des essais. L'urée à la concentration $\mathrm{M}$ a donc été systématiquement incluse dans les milieux de broyage.

\section{Virus dans les racines}

Pendant la période hivernale et jusqu'en avril, le virus a pu être détecté sur un petit nombre de cultivars et à des concentrations très faibles par rapport à celles que l'on peut mettre en évidence dans des feuilles infectées en été (tabl. 3). Les analyses effectuées sur 
TABLEAU 3

Détection en ELISA du VMB dans les racines de différents cultivars de blé à 3 époques d'une culture de blés d'hiver. La D.O. correspond à la somme de 2 répétitions (au niveau de la plaque) d'un échantillon de 10 plantes. Les témoins racines saines proviennent du cv. « Hardi » cultivé à Versailles. Aucune différence importante de bruit de fond n'a pu être observée lors de l'analyse en ELISA de II cv. différents de blés sains. Les extraits racinaires sont dilués 15 fois et les extraits foliaires de plantes saines et infectées utilisés comme marqueur ELISA sont dilués 1000 fois. Detection of WSBMV by ELISA in roots by winter wheat cultivars at three stages of development. Healthy root controls were prepared from cv. "Hardi » grown at Versailles. No positive WSBMV reaction was observed by ELISA with healthy roots of the other cultivars tested. Root and leaf extracts were diluted 15 and 1000 times respectively.

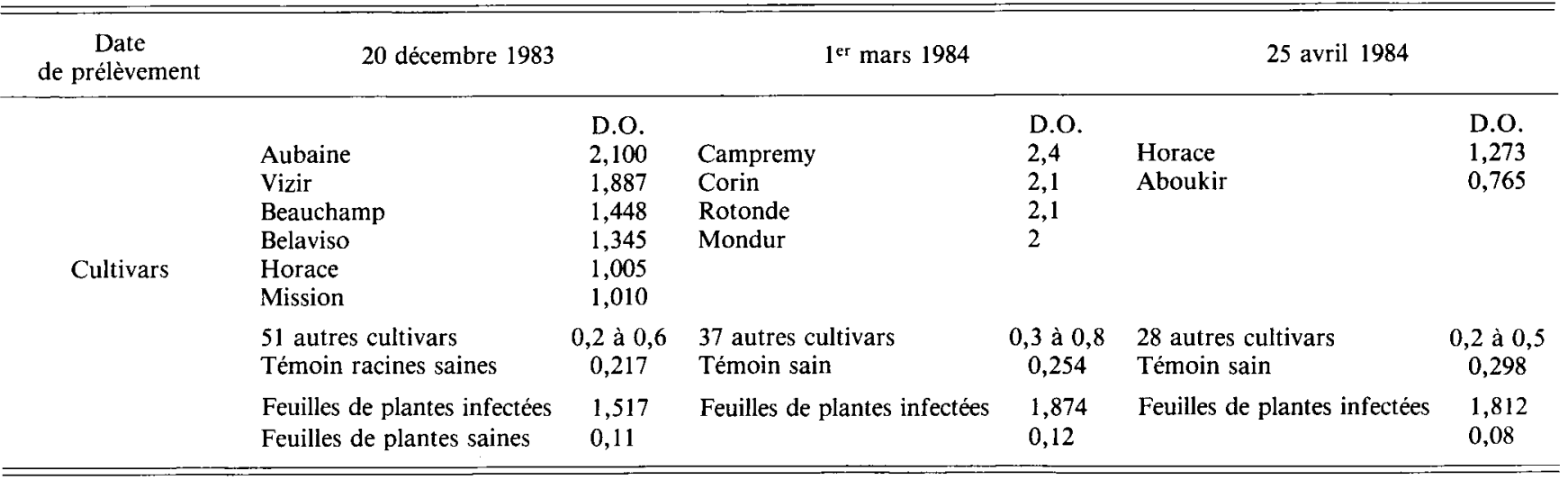

des échantillons prélevés postérieurement sont encore moins discriminantes: les D.O. en ELISA s'étalent sur une échelle réduite entre les témoins sains et les échantillons de cultivars présentant ou non des symptômes (fig. 1). Cependant, la plupart des cultivars sensibles présentent des D.O. supérieures à celles des cultivars résistants au champ.

\section{Virus dans les feuilles}

Les résultats des essais de détection du virus effectués avant l'apparition des symptômes de mosaïque sont en général négatifs. A la fin du mois de mai, pour des lots de feuilles prélevées au hasard provenant de talles bien développées, seuls 11 échantillons sur 18 correspondant à 8 cultivars sensibles réagissent positivement en ELISA (fig. 2 ). Les résultats négatifs correspondent à des cultivars caractérisés par une fréquence faible de plantes exprimant des symptômes ou à "Talent ». La recherche de l'antigène a été alors effectuée sur de jeunes talles secondaires pour lesquelles les symptômes de mosaïque sont plus fréquents.

Ayant enregistré des D.O. assez voisines pour les différents étages foliaires des jeunes talles avec symptômes, les échantillons sont donc constitués par les dernières feuilles de 10 jeunes talles de 10 plantes différentes.

Bien que ce type d'échantillonnage ne permette pas de distinguer entre une faible concentration en virus dans la plupart des talles ou une forte concentration dans un petit nombre de talles, il a été retenu afin de réduire les conséquences d'une hétérogénéité éventuelle des contaminations. L'antigène viral a été recherché pour 13 cultivars sans symptôme et parallèlement pour 4 cultivars̄ exprimant les symptômes de mosaïque avec des fréquences très faibles à fortes (tabl. 4). Tous les cultivars sensibles, quelle que soit leur réaction symptomatologique, présentent des concentrations élevées en antigène viral. Les cultivars sans symptôme peuvent être regroupés provisoirement en 3 classes. La classe B est la mieux caractérisée par une concentration faible en antigène viral reproduite dans au moins 2 essais distincts. Les classes $\mathrm{A}$ et $\mathrm{AB}$ sont constituées par des cultivars pour lesquels l'analyse virale a porté soit sur un seul échantillon, soit sur plusieurs échantillons (cas de " Fidel »), mais sans que l'on ait enregistré en ELISA des D.O. supérieures au double de celle du bruit de fond moyen des témoins sains.

\section{Analyse comparative des populations de 4 types d'organismes colonisant les racines de différents cultivars de blé}

Cette étude a porté sur 12 cultivars sans symptôme et 5 cultivars présentant des symptômes de mosaïque avec une fréquence très faible : (FD 83043), faible : (H 12), «Vizir », (FD 82038) ou forte : «Pursang » (tabl. 5).

L'analyse des sommes de fréquence des populations de ces 4 types d'organismes permet de proposer un classement en 3 groupes des cultivars :

- faiblement colonisés : " Cargo », « Fleuron", (26.3.10), (H 12),

- nettement colonisés : "Fidel », « Sabre », " Avalon », "Capitole », " Cocagne ", " Albatros », (FD 83038), " Apexal », (CWW 1335/2),

- fortement colonisés (FD 83043) : «Pursang », « Tomclair », « Vizir ».

On note un certain lien entre la fréquence de Polymyxa et celle de l'ensemble des 4 types d'organismes dénombrés. Si cette hypothèse était vérifiée, on pourrait supposer l'existence d'une réaction de résistance non spécifique chez certains cultivars et une absence d'antagonisme marqué entre ces 4 types d'organismes. Plusieurs cultivars semblent résistants à une colonisation par Polymyxa si l'on en juge par l'absence ou le nombre réduit des cystosores détectés. 
TABLEAU 4

Détection en ELISA du VMB dans des jeunes talles de cultivars avec ou sans symptômes de mosaïque. Niveau de détection du virus (la D.O. est mesurée après $8 \mathrm{~h}$ d'hydrolyse du substrat $\grave{a} 20^{\circ} \mathrm{C}$ ).

- I Equivalent au témoin sain

- 2 Double du témoin sain

- 3 Triple du témoin sain

- 4 Quadruple du témoin sain

- > 10 Supérieur au décuple du témoin sain.

WSBMV detection by ELISA in young tillers of winter wheat cultivars with or without leaf symptoms. O.D. value measured $8 \mathrm{~h}$ after the beginning of substrate hydrolysis, relative to control.

\begin{tabular}{|c|c|c|c|c|}
\hline Classes de cultivars & Cultivars & $\begin{array}{l}\text { Fréquence des } \\
\text { symptômes de } \\
\text { mosaïque }\end{array}$ & $\begin{array}{l}\text { Niveau de détection } \\
\text { de l'antigène }\end{array}$ & $\begin{array}{l}\text { Nombre d'essais } \\
\text { de détection }\end{array}$ \\
\hline A & $\begin{array}{l}\text { Apexal } \\
\text { Cocagne } \\
\text { Fleuron } \\
\text { Sabre } \\
\text { (CWW 1335/2) }\end{array}$ & & $\begin{array}{l}1 \\
1 \\
1 \\
1 \\
1\end{array}$ & $\begin{array}{l}1 \\
1 \\
1 \\
1 \\
1\end{array}$ \\
\hline AB & $\begin{array}{l}\text { Albatros } \\
\text { Avalon } \\
\text { Cargo } \\
\text { Fidel } \\
\text { Tomclair }\end{array}$ & Aucun & $\begin{array}{l}1,2 \\
1-2 \\
1-2 \\
1,1,1,1,2,2 \\
1,1,-2\end{array}$ & $\begin{array}{l}2 \\
1 \\
1 \\
6 \\
2\end{array}$ \\
\hline B & $\begin{array}{l}\text { Rempart } \\
\text { Capitole } \\
(26.3 .10)\end{array}$ & & $\begin{array}{l}2,3 \\
3,4 \\
4,2,1\end{array}$ & $\begin{array}{l}2 \\
2 \\
3\end{array}$ \\
\hline $\mathrm{C}$ & $\begin{array}{l}\text { (FD 83043) } \\
\text { (H 12) } \\
\text { Vizir } \\
\text { Pursang }\end{array}$ & $\begin{array}{l}\text { très faible } \\
\text { très faible } \\
\text { faible } \\
\text { forte }\end{array}$ & $\begin{array}{l}>10 \\
>10 \\
>10 \\
>10\end{array}$ & $\begin{array}{l}2 \\
1 \\
1 \\
2\end{array}$ \\
\hline
\end{tabular}

TABLEAU 5

Classement des blés en fonction de la fréquence des cystosores de Polymyxa et notation des fréquences de nématodes et des formes de conservation d'Olpidium et de Pythium.

Frequency of Polymyxa graminis cystosori, nematodes and resting stages of Olpidium \& Pythium on different wheat cultivars.

$$
\begin{array}{rlrl}
\text { Echelle }: 0 & =\text { non observé } & \text { Scale }: 0=\text { none } \\
I & =\text { très rare } & I=\text { verv infrequent } \\
2 & =\text { rare } & 2=\text { infrequent } \\
3 & =\text { assez rare } & 3 & =\text { quite infrequent } \\
4 & =\text { peu fréquent } & 4 & =\text { not very frequent } \\
5 & =\text { assez fréquent } & 5 & =\text { quite frequent }
\end{array}
$$

\begin{tabular}{lcccc}
\hline \multicolumn{1}{c}{ Cultivars } & Polymyxa & Olpidium & Pvthium & Nématodes \\
\hline Cargo & 0 & 1 & 2 & 1 \\
\hline$(26.3 .10)$ & 0 & 1 & 3 & 2 \\
\hline (H 12) & 1 & 1 & 4 & 1 \\
\hline Cocagne & 2 & 2 & 4 & 1 \\
Fidel & 2 & 1 & 4 & 1 \\
Fleuron & 2 & 0 & 2 & 0 \\
(CWW 1335/2) & 2 & 2 & 3 & 1 \\
(FD 83038) & 2 & 3 & 4 & 3 \\
\hline Pursang & 3 & 2 & 5 & 5 \\
Sabre & 3 & 0 & 3 & 3 \\
Tomclair & 3 & 3 & 4 & 1 \\
Vizir & 3 & 2 & 4 & 2 \\
\hline Avalon & 4 & 1 & 3 & 1 \\
Apexal & 4 & 2 & 2 & 3 \\
(FD 83043) & 4 & 3 & 5 & 2 \\
\hline Capitole & 5 & 2 & 3 & 0 \\
Albatros & 5 & 1 & 4 & 1 \\
\hline \hline
\end{tabular}

\section{CONCLUSION}

De grandes différences de sensibilité au virus de la mosaïque du blé sont observées chez les blés. Un nombre important de blés, 14 sur 57 étudiés (dont 11 sont inscrits au catalogue français) n'expriment aucun symptôme apparent au champ en conditions fortement contaminantes. Le comportement de "Talent» n'a pas été clairement élucidé. L'hétérogénéité qualitative et quantitative de l'inoculum viral, dans certaines parcelles de comportement, semble plus vraisemblable qu'une hétérogénéité du génotype de ce cultivar vis-àvis du VMB.

Les 11 blés tendres français dont la généalogie est connue sont d'origine variée (tabl. 6). On remarque cependant parmi leurs géniteurs la présence constante de "Cappelle" ou d'un ou plusieurs de ses ascendants. Le comportement de l'ensemble des géniteurs directs de ces blés tendres ou durs est en cours d'étude.

Une $1^{\text {re }}$ approche du niveau de cette résistance au champ a été abordée par la recherche du virus et du Polymyxa en contamination naturelle. Deux facteurs ont limité la portée de ces analyses : la détection immunoenzymatique de l'antigène viral dans les racines est rendue délicate par sa faible concentration et l'existence d'un bruit de fond important dans la période printanière et estivale de la céréale dans cette partie des plantes, malgré la présence de l'urée dans le milieu de broyage. En raison de ces difficultés, les analyses en ELISA des racines de plantes développées n'ont pu être exploitées. L'analyse quantitative de 
TABLEAU 6

Classement provisoire des blés n'exprimant pas de symptômes de la mosaique du blé au champ. Proposed classification of wheat cultivars without WSBMV field symptoms.

\begin{tabular}{|c|c|c|c|c|}
\hline $\begin{array}{l}\text { Niveau de concentration } \\
\text { du VMB (jeunes talles) }\end{array}$ & $\begin{array}{c}\text { Présence du } \\
\text { Polymyxa (Cystosores) }\end{array}$ & $\begin{array}{l}\text { Catégories } \\
\text { proposées }\end{array}$ & Cultivars & Origine généalogique \\
\hline \multirow{3}{*}{ Faible } & Non détectée & 1 & $(26.3 .10)$ & Hardi $\times\left(\mathrm{US}_{(60)} 43 \times\right.$ Prieur $)$ \\
\hline & Fréquente & 2 & Capitole & Cappelle $\times(83 \mathrm{H} \times$ Etoile de Choisy $)$ \\
\hline & Non analysée & $?$ & Rempart & $($ M $50 \times$ B 21) $\times($ Cappelle $\times$ Aisne $)$ \\
\hline \multirow{3}{*}{$\begin{array}{c}\text { Non détecté } \\
\text { ou } \\
\text { très faible }\end{array}$} & Non détectée & 3 & Cargo & $(\mathrm{N} 66 \times 6410) \times(\mathrm{C} 108 \times \mathrm{MGH} 5732) \times$ Major \\
\hline & $\begin{array}{c}\text { Très rare } \\
\text { à } \\
\text { rare }\end{array}$ & 4 & $\begin{array}{l}\text { Cocagne } \\
\text { Fidel } \\
\text { Fleuron } \\
\text { (CWW 1335/2) } \\
\text { (FD 82058) }\end{array}$ & $\begin{array}{l}{[(\text { Blé des Dômes } \times \text { Heine VII }) \times(515)] \times[\text { Champlein }]} \\
\text { (Horizon } \times \text { Frontana) } \times(\text { Capitole } \times \text { Major }) \\
\left.(\text { Champlein } \times \text { Gaines }) \times \text { (Prieur } \times \text { US }_{(60)} 43\right) \\
(\text { CB } 306 \text { Y70 } \times \text { Maris Widgeon } \times(\text { Hobbit } « \text { SIB } ») \\
\text { Maris Huntsman } \times \text { (Sturdy } \times \text { Sonora })\end{array}$ \\
\hline & $\begin{array}{c}\text { Assez rare } \\
\text { à } \\
\text { assez fréquente }\end{array}$ & 5 & $\begin{array}{l}\text { Tomclair } \\
\text { Sabre } \\
\text { Avalon } \\
\text { Apexal } \\
\text { Albatros }\end{array}$ & $\begin{array}{l}1211 \times 621 \\
(\mathrm{RPB}) \\
\text { TJB 30/148 } \times \text { TL } 365 \mathrm{~A} / 34 \\
(\text { NS } 937 \times \text { Argelato) } \times \text { Capitole } \\
\text { Norda } \times \text { Gaillard }\end{array}$ \\
\hline
\end{tabular}

Polymyxa est elle-même délicate en raison de l'hétérogénéité des contaminations racinaires même chez les cultivars pour lesquels on observe une fréquence élevée de plantes présentant des symptômes de mosaïque.

On remarque que 2 blés, "Cargo " et (26.3.10), sur 12 résistants au champ au VMB ne présentent pas les formes de conservation de Polymyxa sur leurs racines. Des cystosores sont effectivement observés sur 5 blés exprimant plus ou moins de symptômes. Cependant l'attribution d'une note faible, 1 et 2 respectivement aux blés (H 12) et (FD 83038), même si ceux-ci se caractérisent par des fréquences faibles de symptômes, ne permet pas de considérer les notes attribuées à "Cargo » et (26.3.10) comme définitives.

Jusque-là, de nombreux résultats obtenus pour divers virus n'ont pas permis de caractériser une résistance nette de chaque espèce étudiée vis-à-vis de Polymyxa (KUCHAREK et al., 1974). C'est la $1^{\text {re }}$ fois qu'une absence de Polymyxa, vecteur du VMB, est notée pour des cultivars de blé résistants au champ.

Parmi les hypothèses susceptibles d'expliquer la faible concentration du VMB dans ces cultivars, si l'on ne peut probablement pas évoquer une hétérogénéité de l'inoculum du sol en raison des observations effectuées sur les blés sensibles ou une hétérogénéité génétique telle qu'elle a été décrite pour « Centurk» par MODAWI et al. (1982), il reste qu'une résistance à la multiplication peut être prise en considération. Elle déterminerait alors une tolérance encore plus nette que celle qui a été signalée par BockUS \& NIBLETT (1984) dans des essais en laboratoire limités à des notations symptomatologiques. Ces 3 cultivars ne constituent pas un groupe homogène puisque, chez "Capitole ", Polymyxa est fréquent alors que chez (26.3.10), il n'a pas été retrouvé.

Un ou 2 des 3 géniteurs de (26.3.10) : $\mathrm{US}_{(60)} 43$ ou/et " Prieur » doivent être résistants à la mosaiqque du blé en raison de la forte sensibilité de "Hardi ». Le comportement de ces 2 blés doit être particulièrement analysé ; en effet, s'ils n'assurent pas la forma- tion de cystosores de Polymyxa, ils peuvent favoriser une réduction de l'inoculum du sol.

La catégorie 4 inclut des blés assez résistants au Polymyxa et chez lesquels l'antigène viral n'a pas été retrouvé. Ces cultivars sont probablement résistants au VMB. «Albatros ", «Apexal », « Avalon», "Sabre » et «Tomclair » constituent un groupe de cultivars nettement favorables à la réalisation d'un cycle complet de Polymyxa et peuvent être considérés comme résistants au virus sans que l'on puisse indiquer laquelle des 3 étapes du cycle viral du virus dans la plante est en cause : la multiplication racinaire, la migration vers la partie aérienne de la plante, la multiplication foliaire.

L'existence de cultivars de blés résistants à l'installation de Polymyxa ou à la multiplication du virus permet d'envisager, chez cette céréale, l'existence d'au moins 2 gènes, dont les produits limitent la réalisation du cycle du VMB.

Ces résultats sont différents de ceux obtenus par DUBAY et al. (1970) et MODAWI et al. (1982) qui-supposent l'existence d'un seul gène de résistance au VMB chez le blé. Cependant, l'analyse présentée par ces auteurs porte seulement sur l'observation des symptômes apparus chez les descendants de croisements faisant intervenir respectivement 2 à 4 cultivars résistants au champ.

Des analyses effectuées par SHAALAN et al. (1966) et NAKAGAWA et al. (1959) semblent montrer l'existence d'au moins 2 gènes de résistance particulièrement pour certains blés.

Parmi les 12 blés tendres sans symptôme, 5 d'entre eux, dont 4 actuellement inscrits au catalogue français, appartenant aux catégories 3 et 4 devraient, si possible, être retenus pour des emblavements en zones contaminées par le VMB. Ces blés ne pourront montrer leur efficacité à réduire la population de vecteurs porteurs de VMB que dans la mesure où d'autres espèces cultivées n'interviennent pas dans le cycle de ce pathogène. 
Ces 5 blés, pour lesquels le VMB n'a pu être mis en évidence dans leurs parties aériennes, montrent que l'on est en présence d'une résistance effective, même si elle est limitée à ces parties du végétal. Ce matériel génétique peut donc être exploité par le sélectionneur. Une analyse plus approfondie du comportement racinaire doit être envisagée dans des conditions contrôlées. Une telle étude permettra, d'une part, un meilleur classement de ces blés, d'autre part, d'aborder les mécanismes de leur résistance au VMB, en particulier dans sa migration dans les parties aériennes des plantes.

Reçu le 12 septembre 1984. Accepté le 15 février 1985.

\section{REMERCIEMENTS}

Nous remercions vivement MM. Bouchain (ITCF, Blois) et GAR NAUD (G.V.A., Ouzouer-le-Marché) qui ont implanté les essais et mis à notre disposition tout le matériel végétal nécessaire à cette expérimentation.

\section{RÉFÉRENCES BIBLIOGRAPHIQUES}

Bockus W. W., Niblett C. L., 1984. A procedure to identify resistance to wheat soilborne mosaic in wheat seedlings. Plant Dis., 68, 123-124.

Canova A., 1962. Su di una infezione da virus del frumento. Ann. Accad. Agric., Bologna, 73, 291-299.

Clark M. F., Adams A. N., 1977. Characteristics of the microplate method of enzyme linked immunosorbent assay for the detection of plant viruses. J. Gen. Virol., 34, 475-483.

Dubay S. N., Brown C. M., Hooker A. L., 1970. Inheritance of field reaction to soil borne wheat mosaic virus. Crop Sci., 10, 9395.

Gugerli P., 1979. Potato virus A and potato leafroll virus : purification, antiserum production and serological detection in potato and test plants by enzyme linked immunosorbent assay, ELISA. Phytopathol. Z., 96, 97-107.

Kucharek T. A., Walker J. H., Barnett R. D., 1974. Effect of cultivar resistance and soil fumigation on soilborne wheat mosaic virus in Florida. Plant Dis. Rep., 58, 878-881.

Kurcman S., 1981. Research on wheat mosaic disease of wheat observed in Eskisekir province. Bitki Koruma Bülteni, 21, 1-17.

Lapierre H., Hariri D., Bouchain F., Garnaud R., 1984. Presence on wheat soil borne mosaic virus in France. Abstr. 4th Conf. on virus diseases of gramineae in Europe, Braunschweig.
Mc Kinney H. H., 1948. Wheats immune from soil borne mosaic viruses in the field, susceptible when inoculated manually. Phytopathology, 38, 1003-1013.

Matthews R. E. F., 1982. Classification and nomenclature of viruses. Intervirology, 17, 1-199.

Modawi R. S., Heyne E. G., Brunetta P., Willis W. G., 1982. Genetics studies of field reaction to wheat soil borne mosaic. Plant Dis., 66, 1183-1184.

Nakagawa M., Soga Y., Watanabe S., Gocho H., Nishio K., 1959. Genetical studies on the wheat mosaic virus. II. Genes affecting the inheritance of susceptibility to strains of yellow mosaic virus in varietal crosses of wheat. Jpn. J. Breed., 9, 118-120.

Powell C. A., 1976. The relationship between soil borne wheat mosaic virus and tobacco mosaic virus. Virology, 71, 453-462.

Proeseler G., Stanarius A., Eisbein K., 1982. Nachweis weiterer Viren an Getreide in der DDR. Arch. Phytopathol. u. Pflanzenschutz, Berlin, 18, 397-403.

Shaalan M. I., Heyne E. G., Sill W. H. Jr, 1966. Breeding wheat for resistance to soil-borne wheat mosaic virus, wheat streak mosaic virus, leaf rust, stem rust and bunt. Phytopathology, 56, 664-668.

Shirako Y., Brakke M. K., 1984. Spontaneous deletion mutation of soil borne wheat mosaic virus RNA. II. J. gen. Virol, 65, 855-858. Tsuchizaki T., Hibino H., Saito Y., 1975. The biological function of short and long particles of soil borne wheat mosaic virus. Phytopathology, 65, 523-532. 